ler Darstellung der Hauptvalenzen (Fig. 2) deutlich. Bei C und Si gehen die Pfeile nach oben, die Valenzen werden negativ, das Atom bekommt gegenüber Elektronen eine ansaugende Wirkung.

Ebenso in den großen Perioden hinter As, Sb, Bi.
He im innersten Ringe 2 statt 3 Elektronen stehen. Das grundlegende Sehema, welches die Betrachtung der Kurve der Atomvolumina für die Verteilung der Elektronen auf die einzelnen Ringe nahelegt, ist dann das folgende:

\begin{tabular}{|c|c|c|c|c|c|c|c|c|c|c|c|c|}
\hline \multirow[b]{2}{*}{ Ringschluß } & \multicolumn{10}{|c|}{ Nummer. (Quantenzahl) der Ringe } & \multicolumn{2}{|c|}{ Zahl der Elektronen } \\
\hline & 1 & 2 & 3 & a & b & $a$ & $b$ & a & b' & 7 & Insgesamt & $\begin{array}{l}\text { In den Perioden } \\
\text { (Differenz Gegenuiber } \\
\text { der vorhergeh. Reihe) }\end{array}$ \\
\hline $\mathrm{He} \ldots \ldots \ldots$ & 2 & & & & & & & & & & 2 & $2.1^{2}$ \\
\hline $\mathrm{Ne} \ldots \ldots \ldots$ & 2 & 8 & & & & & & & & & 10 & $2.2^{2}$ \\
\hline$A \ldots \ldots \ldots$ & 2 & 8 & 8 & & & & & & & & 18 & $2.2^{2}$ \\
\hline $\mathrm{Kr} \ldots \ldots \ldots$ & 2 & 8 & 8 & 10 & 8 & & & & & & 36 & $2.3^{2}$ \\
\hline $\mathrm{X} \ldots \ldots \ldots$ & 2 & 8 & 8 & 10 & 8 & 10 & 8 & & & & 54 & $2.3^{2}$ \\
\hline $\operatorname{Em} \ldots \ldots$ & 2 & 8 & 8 & 10 & 8 & 10 & 8 & $\begin{array}{c}10 \\
(+14)\end{array}$ & 8 & & 86 & $2.4^{2}$ \\
\hline- & 2 & 8 & 8 & 10 & 8 & 10 & 8 & $\begin{array}{c}10 \\
(+14)\end{array}$ & 8 & 6 & 92 & - \\
\hline
\end{tabular}

Hier, wo mit Annäherung: an die Edelgase Rb, Cs, Em die nahen Gleichgewichtskonfigurationen dem Atom. kraftfeld für Elektronen ansaugende Wirkung geben, die Pfeile in Fig. 2 sich ebenfalls nach oben wenden, beginnt auch in den groBen Perioden nach voriber. gehendem Nachlassen des Anstiegs eine neue Wendung nach oben.

Satz 3 ist durch Herrn Sommerfelds Ausführungen belegt. Auch hier kann eine zweifache Begründung gegeben werden. Wachsender Kernladung ist die $\mathrm{Zu}$. sammenziehung der AtomgröBen vor allem hinter den Maximis bei $\mathrm{Na}, \mathrm{K}, \mathrm{Rb}, \mathrm{Cs}$ und hinter dem nicht bekanniten und wegen seiner abnormen Atomgröbe in unserer Weltdichte nicht existenzfahigen radioaktiven Alkali, d. h. also bei Ca, Sr, Ba, Ra zuzuschreiben. Zweitens aber auch in den grosen Perioden vor As, $\mathrm{Sb}, \mathrm{Bi}$ das Nachlassen des Anstiegs. Hier ist hinter dem Zehner- der Achterring in Bildung legriffen und zeigt dieselbe Tendenz wie der Achterring der kleinen Perioden, nur, entsprechend seiner geringeren, weil nicht rom Edelgasgleichgewicht ausgehenden Bildungsenergie, in abgesehwächtem Maße. So entstehen die eigentimlichen, rhythmisch im Anstieg der groben Perioden wiederkehrenden Buckel vor $\mathrm{A}_{\mathrm{s}}, \mathrm{Sb}$, Bi. Der schwächeren Bildungsenergie des Achterringes der großen Perioden ist es auch zuzuschreiben, daß nicht schon vor seinem Beginn hinter $\mathrm{Ni}, \mathrm{Pd}, \mathrm{Pt}$ ein Anstieg zu bemerken ist. Dem entspricht es auch, daß hier keine nggativen, Elektronen ansaugenden Valenzen in Fig. 2 zu finden sind.

Die Diskussion der obigen drei Sätze wäre damit durchgeführt. Zu bemerken wäre noch, daß die Buckel bei $N$ und $P$ (rot) in den kleinen Perioden durch Satz 3 nicht ohne weiteres gedeutet werden können. Thnen müssen anderweitige Annahmen zugrunde gelegt werden. Te fält ganz aus dem Sehema des Kurvenverlaufs heraus, ebenso wie die Erden. Doçh dürfen Abweichungen von der Norm im Beginn der Atombildung und bei sehr großen Atomen, wo innere Zusammenstïrze und Umordnungen rorkommen mögen, am ehesten erwartet werden.

Endlich noch ein Wort tiber die Annahme der Ring. anordnung, welche der obigen Erörterung zugrunde liegt. Sie geht auf Besonderheiten innerer Umord. nungen, wie sie durch die Arbeiten Debyes, Vegards und anderer nahegelegt sind, nicht ein, läkt also hinter
Man begreift, warum der Doppelring $4, a, b, 5, a, b$ bei Bildung seiner Achterhälfte $b$ in allen KrafteldauBerungen schwächer funktionieren muß als der einheitliche Quantenring der kleinen Perioden. Sowohl der Anstieg wie der Abfall, der nach obigen Sätzen zustande kommt, ist flauer. Das Energiequant, welches dort auf einen einheitlichen Ring entfällt, verteilt sich in den großen Perioden auf einen Doppelring.

Trotz aller Umordnungen, welche sich in das obige Schema einfügen mögen, und welehe insbesondere bei den Erden anzunehmen sind, bleibt seine GesetzmäBigkeit erhalten. Die Atomvolumina bezeugen dies. Die Stabilitut der kinge scheint dabei Gleichheit des Abstandes der einzelnen Ringelektronen in den ersten der kleinen und großen Perioden $z u$ erfordern; denn sowohl die Ringradien wie die Elektronenzahlen nehmen in ihnen wie die Quadrate der Quanten. zahlen zu.

Zusammenfassend können wir sagen, daß unser Verständnis dex Kurve der Atomvolumina durch Überlegungen, wie die obigen, gefördert ist und seine Deutung, wenn auch noch nicht litckenlos, so doch in den Grundzügen bis in thythmisch wioderkehrende Einzelheiten hinein feststeht.

Darmstadt, den 16. Juli 1919.

\section{H. Baerwald}

\section{Die Frscheinungen an einzelnen radioaktiven Probekörpern der Größenordnung $10^{-4}$ bis $10^{-5} \mathrm{~cm}$.}

Der eine' von uns hat in der Abhandlung ,Uber die Teilbarkeit der Elektrizität(t) bereits darauf hingewiesen, das auch hinsichtlich der als $\alpha$ Partikel bezeichnetea Komplexe die kritische Fragestellung über dio Gleichheit dieser Partikel vorerst durch eigene Versuche beantwortet werden müBte, welche iłm nicht mehr außerhalb des Rahmens experimenteller Möglichkeit zu liegen schienen. Seither haben wir die elektrischen Vorgänge an einzelnen radioaktiven Probekörpern der Größeñordnung $10^{-4}-10^{-5} \mathrm{~cm}$ untersucht und quantitativ verfolgt. Wir haben der Wiener Akademie der Wissensehaften am 5. März 1919 ein versiegeltes Schreiben unter dem Motto ,Radioaktivität und Elektrizität" uberreicht, in dem bereits ein

1) Annalen der Physik Bd. 56, 1918; S. 69. 
Teil der grundlegenden Resultate enthalten ist. Eine ausführliche Veröffentlichung erfolgt demnächst,

Wien, Physikalisches Institut, 6. August 1919.

\section{F. Ehrenhaft. D. K. Konstantinousky.}

\section{Bemerkung zu meinem Aufsatze:}

Zur Prüfung der allgemeinen Relativitätstheorie.

In meinem Aufsatze steht, worauf mich Herr Ein. stein aufmerksam macht, ein ganz irrefuhrender Satz, den ich richtigstellen möchte.

Anaf Seite 635. in der vorletzten Zeile der ersten Spalte heiBt es: Die Kenntnis des Gravitationspotentials an der Oberfläche des Sternes, d. h. also die Kenntnis der Gravitationsverschiebung der Spektrallinien, erlaubt das Verhältnis: $\frac{\text { Masse }}{\text { Dichte }}$ für einen Stern zu berechnen: Das ist nicht richtig und steht in Widerspruch zu dem 22 Zeilen vorangehenden Satze, wonach man auf Grund der Kenntnis des Gravitationspotentials an der Oberfläche nur miteinander verträg. liohe Werte für Masse und Dichte zu berechnen vermag. Will man also das Verhältnis: $\frac{\text { Masse }}{\text { Dichte }}$ berechnen, so muß man über eine der beiden Gröben eine Verfügung treffen. Es fehlt folglich in dem betref. fenden Satze ein Nebensatz des Inhaltes, daß iber die mittleren Dichten der B-Sterne verfügt wurde, und dab in der folgenden Rechnung dafür der Wert: $1 / 10$ Sonnendichte gewählt wurde. (Siehe Zeile 14 von unten auf Seite 635 , 1. Spalte.)

Die mittlere Dichte kann man übrigens nur für eine kleine Zahl von B-Sternen abschätzen, die spektroskopische Doppelsterne sind und bei denen man zugleich, infolge periodisch wiederkehrender Bedeckungen beider Komponenten, einen Lichtwechsel beobachtet. Was die Einzelheiten betrifft, so möchte ich auf die demnächst erscheinende ausführliche Publikation in der Phys. Zeitschrift verweisen.

Berlin-Neubabelsberg, den 28. August 1919. Erwin Freundlich.

\section{Gesellschaft für Erdkunde zu Berlin.}

In der Sitzung am 14. Juni 1919 hielt Professor Bergsträßer (Berlin) einen Vortrag mit Lichtbildern uber seine Reisen in Syrien, die er in den Jahren 1914 und $1918 \mathrm{zwecks}$ sprachwissenschaftlicher Studien ausgefuhrt hat. Bei diesen Reisen handelte es sich nicht nur um die Sammlung von sprachlichem Material nach dem Muster des französischen Atlas linguistique, sondern es war auch eine sprachgeographische Erforschung von Syrien geplant, um von den sprachlichen Verhältnissen des ganzen Landes eine Vorstellung zu bekommen und vor allem die Abhängigkeit dialektischer Verschiedenheiten von geographischen, ethnogra. phischen und historischen Bedingungen klarzulegen. Die sprachwissenschaftlichen Ergebnisse der ersten Reise hat der Vortragende in einem 1915 erschienenen Sprachatlas von Syrien und Palustina niedergelegt.

Am geringsten sind die Verschiedenheiten zwischen den Dialekten der größeren Städte; $z a$ diesem verbältnismäBig einheitlichen Städterdialekt steht im schäristen Gegensatz der nicht ganz so einbeitliche, aber immer noch. wenig differenzierte Beduinendialekt; und zwar ist. die Sprache der Beduinen am reinsten arabisch, während die der städter Berührungen mit einer anderen semitischen Sprache, dem Aramäischen zeigt, dessen bekanntester Dialekt das Syrische ist.
Das erklärt sich aus den ethnographisch-historischen Verhältnissen; während die Beduinen einigermaßen unvermischte Araber sind, ist die städtische Bevölkerung hervorgegangen aus einer Mischung zwischen einer ziemlich schwachen Oberschicht von arabischen Eroberern und der alteingesessenen, aramüisch sprechenden Masse. Die Sprache der ansässigen Bevölkerung des flachen Landes bildet, in sich entsprechend der geographischen Gliederung des Landes stark dialektisch differenziert, eine Mittelstufe zwisehen diesen beiden Extremen der St,ädter- und Beduinensprache, und zwar im Norden sich mehr der ersteren, im Süden mehr der letzteren zuneigend. Die Sprache der ansässigen Landbevölkerung zeigt daher die größten Unterschiede gegen diejenige der Stïdter in Jerusalem, also im Süden, gegen diejenige der Beduinen aber in Aleppo, im Norden. Auch darin spiegeln sich die ethnographischen und historischen Verhältnisse: während die Bauernbevölkerung im Norden ebenso wie die stïdtische Bevölkerung überwiegend auf aramüisch sprechende alteingesessene Elemente zurückgeht, ist sie im Süden gröstenteils durch den auch jetzt noch fortdauernden Prozeß des SeBhaftwerdens von Beduinen neu entstanden. Der erwilnten vorarabischen Landessprache, dem Aramäischen, das als die Sprache $\mathbf{P} a$ u stinas zur Zeit Christi und als die Ursprache seiner Worte eine erhöhte Aufmerksamkeit verdient, wurde besondere Sorgialt gewidmet, Aramäisch wird jetzt nur noch in drei Dörfern des Antilibanons, vor allem in Malūla, nördlich von Damaskus gesprochen, aber es nimmt immer mehr von arabischem sprachgut auf, ist daher schon stark rom Arabischen beeinflußt und sicher dazu verurteilt, über kurz oder lang von $\mathrm{ihm}$ verdrängt $\mathrm{zu}$ werden. Wie es dort vielfach üblich ist, wendet sich fast die ganze männliche $\mathrm{Be}$ völkerung MaTūlas demselben Berufe zu. Sie liefert die Bäcker für die Stadt Damaskus.

Die vorgeführten Lichtbilder, die zum Teil nach dem Lumièreschen Autochromverfahren aufgenommen waren, zeigten manche charakteristische Landschaften, vor allem vom Taurus und Libanon, Volkstypen, sowie manche kulturgeographisehe Einzelheiten, wie z. B. die schrïgen Ziegeldächer im nördlichen Teil des Gebietes und die großen zur Bewatsserung dienenden Wasserränder am Orontes, die nieht, wie in Agypten, durch menschliche oder tierische Kraft, sondern durch die StoBkraft des strömenden Flusses in Tätigkeit gesetzt werden.

In der Sitzung am 5. Juli hielt Prof. $F$. Jaeger (Lichterfelde) einen Vortrag: Fünf Krieg- und Forschungsjahre in Deutseh-Suidwest-Afrika. Der Vortragende war mit seinem Assistenten Dr. Waibel Ende Februar 1914 auf dem Dampfer "Gertrud Woermann" nach Südafrika abgefahren, hatte zunächst die südafrikanische Union bereist und ging Ende April von Lïderitzbucht nach Tsumeb, dann nach Windlruk, wo um Pfingsten eine landwirtschaftliche Ausstellung einen schönen und lehrreichen toberblick über die wirtschaftlichen Leistungen des Landes und die Erfolge der dentschen Kolonisation darbot. Ende Juni brach er nach der im nördlichen Gebiet, zu der an der Grenze des Ambolandes gelegenen Etoschapfanne aut, wo ihn die Nachricht vom Ausbruch des Krieges erreichte, den er als Soldat der Sehutztruppe bis zum FriedensschluB von Khorab im Juli 1915 mitmachte. Da nur die aktive Schutztruppe in Aus interniert warde, so durite er mit polizeilicher Erlaubnis im Lande umherreisen und so dessen größten Teil kennen lernen. Die Zu- 